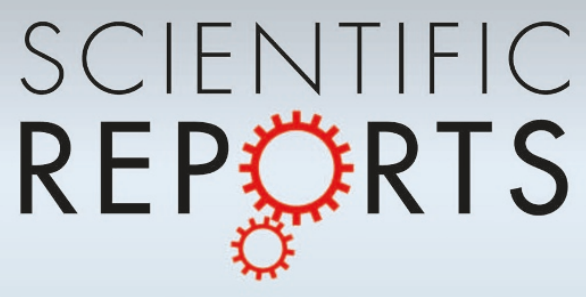

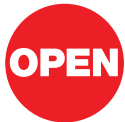

SUBJECT AREAS:

VISUAL SYSTEM

AUDITORY SYSTEM

NEURON

SOMATOSENSORY SYSTEM

Received

13 September 2012

Accepted

8 March 2013

Published

27 March 2013

Correspondence and requests for materials should be addressed to K.I. (kazuol@|su.edu)

\section{Global Hyper-synchronous Spontaneous Activity in the Developing Optic Tectum}

\author{
Kazuo Imaizumi ${ }^{1,3}$, Jonathan Y. Shih ${ }^{2}$ \& Hamilton E. Farris ${ }^{3}$
}

\author{
'Department of Comparative Biomedical Sciences, Louisiana State University School of Veterinary Medicine, Baton Rouge, LA, \\ ${ }^{2}$ Center for Integrative Neuroscience, University of California, San Francisco, CA, ${ }^{3}$ Neuroscience Center, Department of \\ Otorhinolaryngology, Lovisiana State University Health Sciences Center, New Orleans, LA.
}

Studies of patterned spontaneous activity can elucidate how the organization of neural circuits emerges. Using in vivo two-photon $\mathrm{Ca}^{2+}$ imaging, we studied spatio-temporal patterns of spontaneous activity in the optic tectum of Xenopus tadpoles. We found rhythmic patterns of global synchronous spontaneous activity between neurons, which depends on visual experience and developmental stage. By contrast, synchronous spontaneous activity between non-neuronal cells is mediated more locally. To understand the source of the neuronal spontaneous activity, input to the tectum was systematically removed. Whereas removing input from the visual or mechanosensory system alone had little effect on patterned spontaneous activity, removing input from both systems drastically altered it. These results suggest that either input is sufficient to maintain the intrinsically generated spontaneous activity and that patterned spontaneous activity results from input from multisensory systems. Thus, the amphibian midbrain differs from the mammalian visual system, whose spontaneous activity is controlled by retinal waves.

$\mathrm{n}$ the developing brain, spontaneous activity plays an important instructive role in topographic organization ${ }^{1,2}$, as neurons with correlated spontaneous activity have connectivity with similar functional and physiological features $^{3-5}$. There is, however, some variance in the development of correlated spontaneous activity. In mammalian neocortex, highly correlated spontaneous activity in a large neuronal population at an early age is subsequently replaced by less correlated activity in sparse populations ${ }^{6}$. In the zebrafish spinal cord, on the other hand, sporadic spontaneous activity in a small population of cells is switched to tightly correlated activity across the larger population within a few hours during the early developmental stage ${ }^{7}$. These opposite trends in activity in the different systems (sensory and motor) or different developmental stages suggest that, to understand the development of precise topographic organization, it is crucial to understand spatio-temporal patterns of spontaneous activity in the developing brain. We addressed this question in the developing optic tectum of albino tadpoles, Xenopus laevis, using an in vivo two-photon $\mathrm{Ca}^{2+}$ imaging technique. The Xenopus tectum has been an important model system for the study of developmental plasticity of retinotectal organization ${ }^{8-11}$ and, more recently, of multisensory integration ${ }^{12}$. However, study of spontaneous activity in the tectum has been largely overlooked. Only a recent study using an in vitro $\mathrm{Ca}^{2+}$ imaging technique addressed this question, and found sporadic spontaneous activity ${ }^{13}$. However, spatio-temporal patterns of spontaneous activity in the intact tectum are not yet understood. Spontaneous activity in developing brain circuits often originates from more than one source: self-generating activity ${ }^{14,15}$ and/or ascending input ${ }^{2}$. In a recent study, acute eye enucleation in mouse revealed only a partial influence on synchronous spontaneous activity in primary visual cortex before eye opening $^{16}$, leaving the question open of what the origin for the residual activity could be. In the current study, thus, we separately manipulated and measured both self-generated activity and ascending input in the developing midbrain to define both the nature and the source of the spatio-temporal patterns of spontaneous activity in the tectal neurons during development.

\section{Results}

The optic tectum in Xenopus laevis tadpole is roughly divided into three layers: the neuropil that mainly consists of ascending sensory axons and tectal dendrites, the cell body layer that consists of tectal somata, and the ventricular layer that contains radial glial "and other non-neuronal" cells (Fig. 1A, S1). Thus, the layers delineate the input (the neuropil) and output (somata) regions in the tectum. To assess spontaneous activity in these layers, we used in vivo two-photon $\mathrm{Ca}^{2+}$ imaging of both the neuropil and somata stained by a cell-permeant intracellular calcium indicator, Oregon Green BAPTA-1 AM (OGB1-AM), in 50 tecta (Fig. 1A, S1) ${ }^{17}$. A slow frame speed (0.6 frames/s, fps) allowed us to capture the spontaneous events while minimizing the chance of photo damage. 
Because visual experience enhances the growth of tectal dendrites in Xenopus tadpoles ${ }^{18}$, we hypothesized that visual experience would alter spontaneous activity patterns in the developing midbrain of optic tectum. To test this hypothesis, tadpoles were raised in one of two different conditions (control 12 hours light/dark or 24 hours dark).

Spontaneous $\mathrm{Ca}^{2+}$ events in the input layer of the tectum. Figure $1 \mathrm{~B}$ illustrates six representative $\mathrm{Ca}^{2+}$ traces of spontaneous activity in the input layer of the neuropil at different developmental stages in the two different rearing conditions. We imaged 9 and 4 tecta (CTRL vs. Dark) at stage 45/46, 5 and 11 tecta at stage 47/48, and 10 and 11 tecta at stage 49/50. At stage 45/46, event frequency $\left(\mathrm{Ca}^{2+}\right.$ events/min) and magnitude $(\Delta \mathrm{F} / \mathrm{F})$ of spontaneous activity were low and small (Fig. $1 \mathrm{~B}$ top panels). At later stages, they developed a higher event frequency and a larger event magnitude of spontaneous activity (Fig. 1B middle and bottom panels). Contrary to our hypothesis, within-stage comparisons revealed no significant difference in the event frequency (unpaired $t$-test: $p=$ 0.06 at stage $45 / 46 ; p=0.88$ at stage $47 / 48$; and $p=0.69$ at stage $49 /$ 50 ) and magnitude (unpaired $t$-test: $p=0.55$ at stage $45 / 46 ; p=0.28$ at stage $47 / 48$; and $p=0.07$ at stage $49 / 50$ ) of spontaneous activity between the two different rearing conditions (Fig. 1C, D left panels). However, spontaneous $\mathrm{Ca}^{2+}$ events were stage-dependent (Fig. 1C, D right panels). Both event frequency and magnitude were the highest at stage $47 / 48$. Event magnitude increased significantly from stage $45 / 46$ to $47 / 48$, and decreased significantly to stage $49 / 50$.

A rhythmic pattern is a characteristic feature of spontaneous activity in the developing brain ${ }^{14}$. To quantify the development of temporal patterns of $\mathrm{Ca}^{2+}$ events, we computed the coefficient of variation $(\mathrm{CV})$ of inter-event intervals (IEIs): SD divided by the mean IEI. Here, the smaller the CV, the more periodic the IEIs. There was no significant difference (unpaired $t$-test: $p=0.78$ at stage $45 / 46$; $p=0.22$ at stage $47 / 48$; and $p=0.35$ at stage $49 / 50$ ) in the CV of IEIs between the two rearing conditions (Fig. 1E left panel). More importantly, the CV of IEIs showed no significant difference between the stages (Fig. 1E right panel), indicating that spontaneous $\mathrm{Ca}^{2+}$ events maintained consistent rhythmic patterns across developmental stages. These rhythmic patterns displayed intervals of 15-25 s (Fig. 1C). Overall, the frequency and magnitude of spontaneous $\mathrm{Ca}^{2+}$ events in the neuropil depended on stage, not visual-experience, and spontaneous activity had consistent rhythmic patterns throughout the stages tested. These results in the input layer led us to test whether spontaneous $\mathrm{Ca}^{2+}$ events in the output layer show similar dependencies.

Spontaneous $\mathrm{Ca}^{2+}$ events in the output layer of the tectum. Using the same 50 tecta, we examined spontaneous $\mathrm{Ca}^{2+}$ events in the output layer in regions of interest (ROIs) that were conservatively selected on clearly distinguishable somata. This allowed us to perform between-cell analysis of spontaneous activity, an analysis we were unable to perform with the layer-wide measurements of the neuropil. Figure 2A, B illustrates the occurrence of spontaneous $\mathrm{Ca}^{2+}$ events and representative $\mathrm{Ca}^{2+}$ traces in single somata (black) and neuropil (red), respectively. Synchronized spontaneous activity was evident in both soma-soma and soma-neuropil pairs. We computed cross-correlation functions (CCFs) for all pairs (Fig. 2C) because visual experience may enhance recurrent activity ${ }^{19}$. We found that many had high maximum correlation coefficient (MCC) values. Such high MCC values of $\sim 0.5$ indicate hypersynchronous spontaneous activity. The percentage of cell pairs in which the MCC occurred at $0 \mathrm{~s}$ time lag (hereafter, $0 \mathrm{~s}$ lag MCC) was 83.1 ( \pm 20.7; mean \pm S.D.), in 50 tecta (Fig. 2C insets). However, the high prevalence of $0 \mathrm{~s}$ lag MCCs could have been an artifact caused by the slow frame speed of $0.6 \mathrm{fps}$. We tested this possibility using faster frame speeds of 10-100 fps for a subset of eight tecta (nine imaging sessions). Four out of nine fast imaging sessions used $20 \mathrm{fps}$ and the rest were 10, 17, 33, 42, and $100 \mathrm{fps}$. The test showed that even at this high frame speed, extreme synchrony was still evident, albeit reduced (Fig. 3). Whereas $0 \mathrm{~s}$ lag MCCs occurred in $52.7 \%( \pm 31.5)$ of cell pairs for fast frame imaging
A

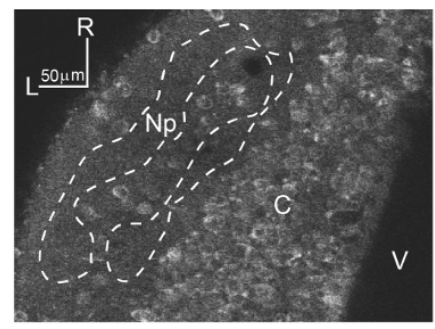

C

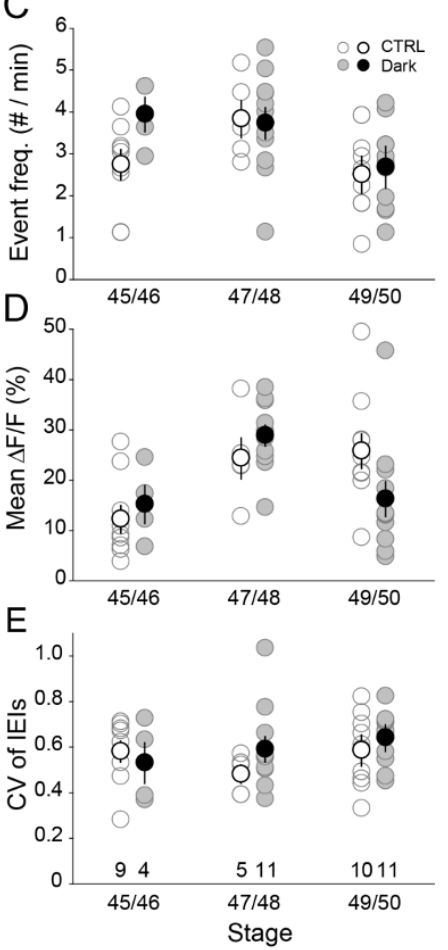

B
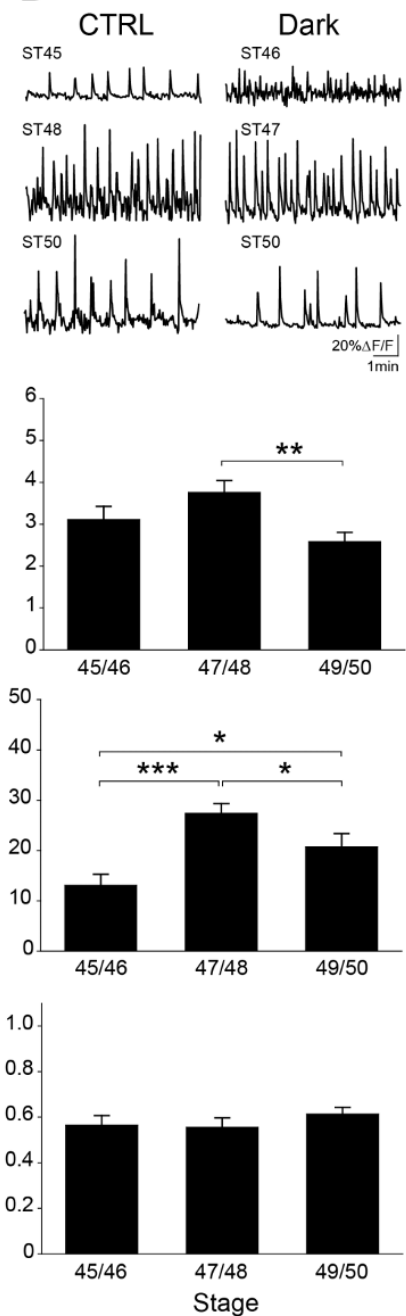

Figure 1 | Development of spontaneous $\mathrm{Ca}^{2+}$ events in Xenopus tectum depends on stage but not visual-experience. (A) Movie frame of optic tectum stained by OGB-1AM. Spontaneous $\mathrm{Ca}^{2+}$ events were assessed in the neuropil (Np; surrounded by a dashed line). C: cell body layer, V: ventricular layer, R: rostral, L: lateral. (B) Example traces of spontaneous $\mathrm{Ca}^{2+}$ events in tecta reared under a control 12/12 hours light/dark cycle (CTRL: left column) and a dark condition (Dark: right column) at different developmental stages. (C-D) Analysis of event frequency and magnitude of spontaneous activity. Within-stage comparisons between the two rearing conditions were performed by unpaired $t$-test (left column). Between-stage comparisons after pooling the two rearing conditions were performed by Fisher's protected least significant difference test (right column). (C) Mean \pm S.D. of event frequency in each groups is; $2.7 \pm 1.0$ (CTRL) vs. $3.9 \pm 0.8$ (Dark) at stage $45 / 46$,

$3.8 \pm 1.0$ vs. $3.7 \pm 1.2$ at stage $47 / 48$, and $2.5 \pm 1.0$ vs. $2.7 \pm 1.0$ at stage $49 /$ 50. (D) Mean \pm S.D. of event magnitude in each group is; $12.2 \pm 8.1$ (CTRL) vs. $15.1 \pm 7.6$ (Dark) at stage $45 / 46,24.3 \pm 9.1$ vs. $28.9 \pm 6.8$ at stage $47 / 48$, and $25.8 \pm 10.8$ vs. $16.2 \pm 11.4$ at stage $49 / 50$. (E) Coefficient of variation (CV) of inter-event intervals (IEIs) of spontaneous activity. Mean \pm S.D. of CV of IEI in each group is; $0.56 \pm 0.14$ (CTRL) vs. $0.53 \pm 0.18$ at stage $45 / 46,0.48 \pm 0.08$ vs. $0.59 \pm 0.18$ at stage $47 / 48$, and $0.59 \pm 0.15$ vs. $0.64 \pm 0.12$ at stage $49 / 50$. Sample size at each stage in the two different rearing conditions is noted in (E). *: $p<0.05, * *: p<0.01$, $* * *: p<0.001$. Error bars are S.E.M. 

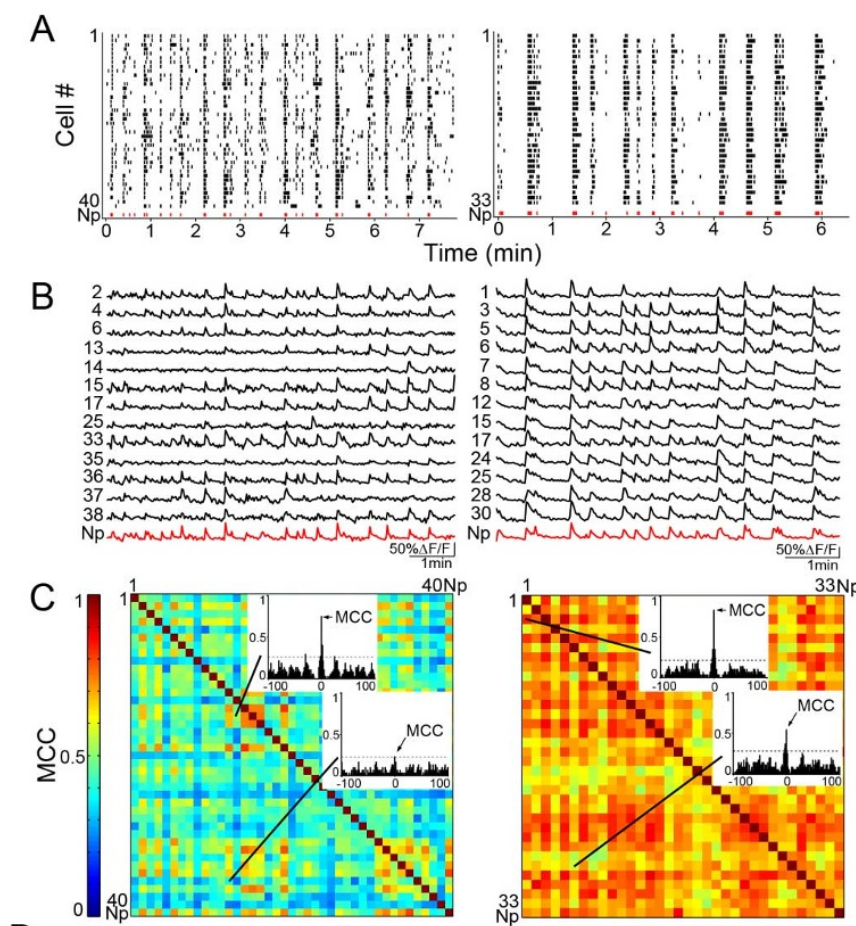

D
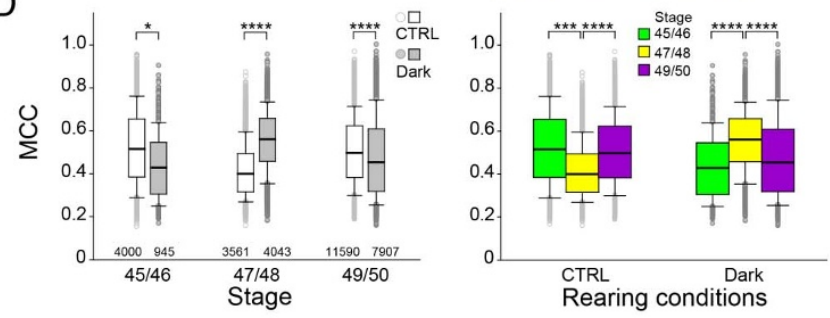

Figure 2 Experience-dependent synchrony in spontaneous $\mathrm{Ca}^{2+}$ events between neurons. (A-C) Examples from two tecta at stage 48 that varied in spontaneous $\mathrm{Ca}^{2+}$ event synchrony (left column: control light/dark; right column: dark-reared). (A) Raster plots of spontaneous $\mathrm{Ca}^{2+}$ events in single neurons and the neuropil ( $\mathrm{Np}$ ) illustrated by black and red dots, respectively. (B) Example traces of 13 single neurons and the neuropil shown in (A). (C) Matrix of maximum correlation coefficient (MCC) values for all cell pairs (including the neuropil) in cross correlation functions (CCFs). Insets: Examples of CCFs. Peak is MCC. A dashed line is $99 \%$ confidence threshold of CCFs. Time scale is between $-100 \mathrm{~s}$ and 100 s. Medians of MCC values: 0.41 (left), 0.71 (right). (D) Comparisons of MCC values under different rearing conditions and at different stages. Box plots illustrate medians (thick horizontal lines), quartiles (box heights), 10th and 90th percentiles (error bars), and the outliers (circles). Statistical comparisons were performed by a resampling test. Pair numbers are illustrated below each distribution (bottom left). For multiple comparisons (right), $p$ values were adjusted by Bonferroni correction. *: $p<0.05, * * *: p<0.001, * * * *: p<0.0001$.

(e.g., Fig. 3B insets), 95.1\% ( \pm 4.5 ) of pairs had $0 \mathrm{~s}$ lag MCCs at the slow frame imaging speed ( $p<0.001$, paired $t$-test; same tecta). All data were pooled because we did not find any correlation between frame speed and the percentage of neuron pairs with $0 \mathrm{~s}$ lag MCCs (a linear regression, $\left.r^{2}=0.02, p>0.05\right)$. Although, the proportion of cells with a 0s lag MCCs may be biased by the slow frame speed, the high proportion (53-83\%) of cells with a 0 s lag MCC strongly suggests that tectal cells receive input from a common source to generate spontaneous activity.

Unlike in the input layer of the tectum, visual experience influenced spontaneous $\mathrm{Ca}^{2+}$ events in the output layer, as MCC values varied within each stage (Fig. 2D left panel), and across developmental stages (Fig. 2D right panel). In general, MCC values were higher in the control than in the dark-reared condition, suggesting that visual experience enhanced synchronized spontaneous activity between cells. However, at stage 47/48, MCC values were lower in the control than in the dark-reared condition. With respect to effects of developmental stage, MCC values at stage 47/48 were significantly lower or higher than at other stages in the control or dark condition, respectively (Fig. 2D right panel). These differences could be caused by differences in neural transmission efficacy from the input (the neuropil) to the output layer (somata). The neural transmission efficacy was defined by synchrony of spontaneous $\mathrm{Ca}^{2+}$ events between the neuropil and somata and assessed by $\mathrm{MCC}$ values in CCFs. However, there was no significant difference of average MCC values between rearing conditions within stage (Fig. 4 left panel) or across developmental stages (Fig. 4 right panel). Taken together, spontaneous $\mathrm{Ca}^{2+}$ events in the output layer (cellcell interactions) differ from those in the input layer (the neuropil), as visual experience and developmental stage influence cell-cell synchronous interactions. Furthermore, these synchronous spontaneous $\mathrm{Ca}^{2+}$ events occur globally in the tectum.

A source of spontaneous $\mathrm{Ca}^{2+}$ events. Based on the results above, it is clear that the frequency and magnitude of spontaneous $\mathrm{Ca}^{2+}$ events in the input layer of the tectum do not covary with the development of cell-cell interactions in the output layer. This, along with the high proportion of cell pairs with $0 \mathrm{~s}$ lag MCC (53-83\%), leads us to hypothesize that the spontaneous activity across somata was selfgenerated. To test this hypothesis, we directly manipulated input from the sensory systems to the tectum. In the optic tectum, visual input by retinal ganglion cells via retinotectal projections from the contralateral eye drives sensory response $\mathrm{e}^{20,21}$. In 10 tecta ( 8 tecta at stage $49 / 50$ and 2 at stage 47/48), following two or three control imaging sessions, we removed visual input to the tectum by enucleating the contralateral eye and subsequently repeated imaging sessions. ROIs were chosen in a large area including the cell body layer and the neuropil. Figure 5A illustrates two examples of spontaneous $\mathrm{Ca}^{2+}$ traces before and after the manipulation. The manipulation altered neither the event frequency nor the event magnitude of spontaneous activity (Fig. 5B, C). Therefore, input from the visual system is not necessary to generate spontaneous activity. The optic tectum also receives mechanosensory input from auditory, somatosensory, and lateral-line systems via the hindbrain (hindbrain-tectal projections $)^{22,23}$. In nine additional tecta (7 tecta at stage 49/50 and 2 at stage 47 ), we removed input from the mechanosensory systems by cutting the connection between the tectum and the hindbrain, while the visual system was intact. This manipulation had only a small impact on event frequency, but no significant effect on event magnitude (Fig. 5E-G). In 11 additional tecta (9 tecta at stage 49/ 50 and 2 at stage 47), we removed input from both visual and mechanosensory systems by enucleating the contralateral eye and cutting the hindbrain-tectal connection. The manipulations drastically and significantly reduced the event frequency and magnitude of the spontaneous activity (Fig. 5I-K): spontaneous activity was persisted in nine tecta but was completely abolished in two others. These results suggest not only that there is some intrinsic (selfgenerating) spontaneous activity within the tectum, but also that input from either sensory system is sufficient to maintain intrinsic spontaneous activity.

The manipulation experiments also tested whether rhythmic activity in an intact condition (Fig. 1C, E) is self-generated. Here, we computed the CV of IEIs before and after the manipulations. Removing input from either the visual or mechanosensory system alone did not alter the CV of IEIs (Fig. 5D, H). However, removing input from both systems significantly increased CVs of IEIs of spontaneous activity for the nine tecta in which spontaneous events were not abolished (Fig. 5L), indicating less rhythmic patterns. These 
A

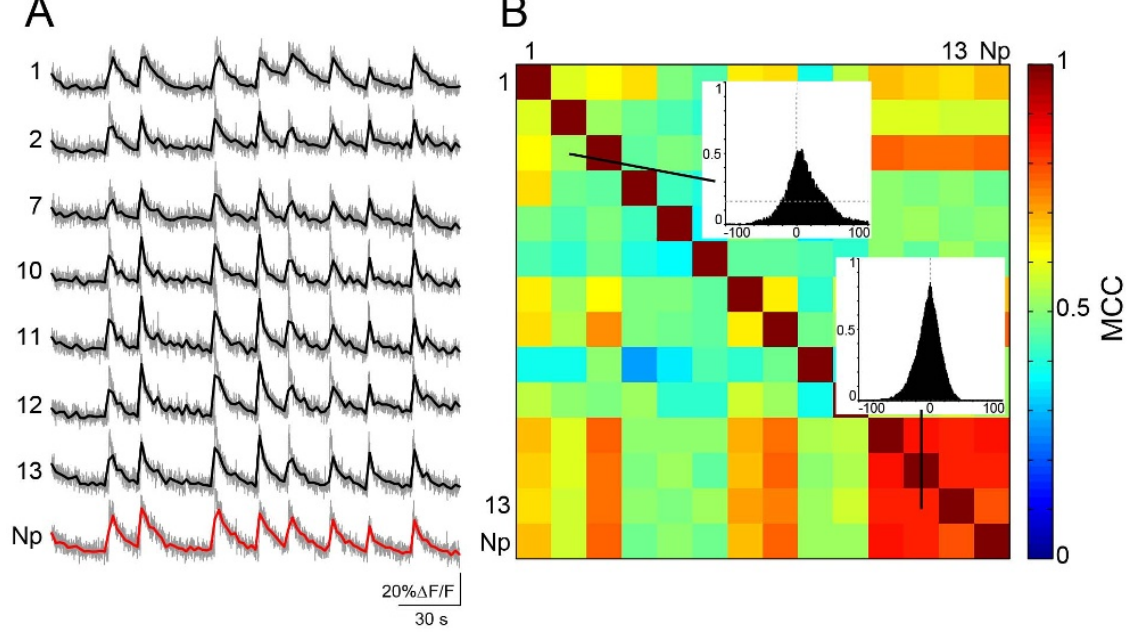

Figure 3 Synchronized spontaneous activity between neurons. $\mathrm{Ca}^{2+}$ imaging was made by a faster frame speed (20 fps). (A) Ca ${ }^{2+}$ traces in seven neurons and the neuropil (Np). Gray lines are raw traces. Black and red lines are running average traces for illustration. (B) Maximum correlation coefficient (MCC) values for all pairs in cross correlation functions (CCFs) in a matrix. Cells are approximately aligned based on the distance along one direction. Inserts: Examples of CCFs for two pairs. Horizontal dashed lines illustrate the $99 \%$ of confidence threshold. A vertical dashed line is at a 0 s time lag.

results strongly suggest that first, the rhythmic patterns of synchronous spontaneous activity are not self-generated within the tectum; and second, input from visual or mechanosensory systems is sufficient to produce these rhythmic patterns.

Local non-neuronal interactions contrast global neuronal synchronization. Our finding of highly synchronized spontaneous activity by tectal neurons is novel. We next addressed whether the same type of synchronization exists for other cells in the tectum. In the ventricular layer, there are radial glial cells (neural progenitor cells), immature neurons, and epithelial cells (Fig. 6A) ${ }^{24,25}$. For four tecta at stage 49/50 (three reared in light/dark and one in dark), we were able to stain both non-neuronal cells and tectal neurons with OGB1-AM (Fig. 6A). Figure 6B illustrates the occurrence of spontaneous $\mathrm{Ca}^{2+}$ events in 62 tectal neurons (gray), neuropil (red) and 61 non-neuronal cells (black and blue; two colors are used later to illustrate different sampling populations) from a single tectum. In contrast to the global neuronal synchronization (gray dots, also see Fig. 2A), there was no such indication for the spontaneous $\mathrm{Ca}^{2+}$ events in non-neuronal cells (black and blue dots). Furthermore, the events in non-neuronal cells often lasted on the
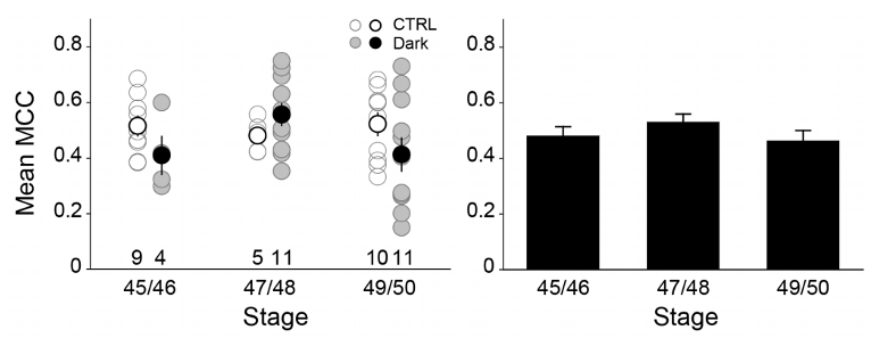

Figure $4 \mid$ Neural transmission efficacy from the input (the neuropil) to the outpur (somata) layer is computed by mean maximum correlation coefficient (MCC) between the neuropil and cells. Within-stage comparisons between two rearing conditions were performed by unpaired $t$-tests (left column). Between-stage comparisons after pooling the two rearing conditions were performed by Fisher's protected least significant difference test (right column). Error bars are S.E.M. No significant difference suggests efficient neural transmission regardless of rearing conditions and developmental stages. Sample size at each stage in the two different rearing conditions is noted (left panel). order of a minute, much longer than the event duration observed in neurons.

We computed CCFs in the neurons (including the neuropil) and the non-neuronal cells separately (Fig. 6C). Within the same tecta, neuronal pairs had higher MCC values than non-neuronal pairs (medians, 0.56 for 6742 neuron pairs vs. 0.40 for 2723 non-neuronal pairs in the four same tecta, $p<0.0001$, resampling test, see Methods). Thus, we did not find the same type of global synchronous activity in non-neuronal cells. However, some traces suggest that there may be more local synchronization in non-neuronal cells. For the $\mathrm{Ca}^{2+}$ traces illustrated in Figure 6D (2 neurons, the neuropil, and 12 non-neuronal cells), neurons (gray) and neuropil (red) were typically similar to each other, but there may be groups of similar clustering activity in non-neuronal cells (black and blue). Although non-neuronal cell traces from different topographic locations (non-neuronal cells $1,11,21,31$, and 41) were dissimilar, non-neuronal cells (blue) from a similar region (filled blue circles in Fig. 6A) had similar or complementary trace shapes. Thus, we analyzed the relative timing of the interactions between non-neuronal cells 55-61 (corresponding to a local clustering in the bottom right corner of Fig. $6 \mathrm{C}$ bottom) to assess local interactions (Fig. 6E). Based on CCFs, Figure 6E illustrates a schematic interaction chart. When two non-neuronal cells had a peak at $0 \mathrm{~s}$ time lag in CCFs, they likely shared a common input source (e.g., $61=60$ ). When they had two equal peaks on both positive and negative time lags, they most likely had a reciprocal interaction (e.g., $58 \leftrightarrow 61$ ) When they had a larger peak at either a positive or negative time lag, they had a dominant interaction (e.g., $59 \leftarrow 55$, $56 \rightarrow 55$ ). We considered peaks occurring within $50 \mathrm{~s}$ lag (see Methods). Within a local cluster, all seven non-neuronal cells had either a common input, reciprocal, or dominant interaction. These results suggest complex local interactions between non-neuronal cells in contrast to the simple global synchronization between neurons in the developing optic tectum.

\section{Discussion}

Spatio-temporal patterns of synchronously active cells are critical to many aspects of neural processing ${ }^{26-29}$ including the development of topographic neural circuits ${ }^{1,2}$. Our study examined such circuitry in the Xenopus optic tectum. To understand the development of neural circuits in the intact animal, we employed in vivo two-photon $\mathrm{Ca}^{2+}$ imaging to simultaneously monitor the spatio-temporal patterns of 


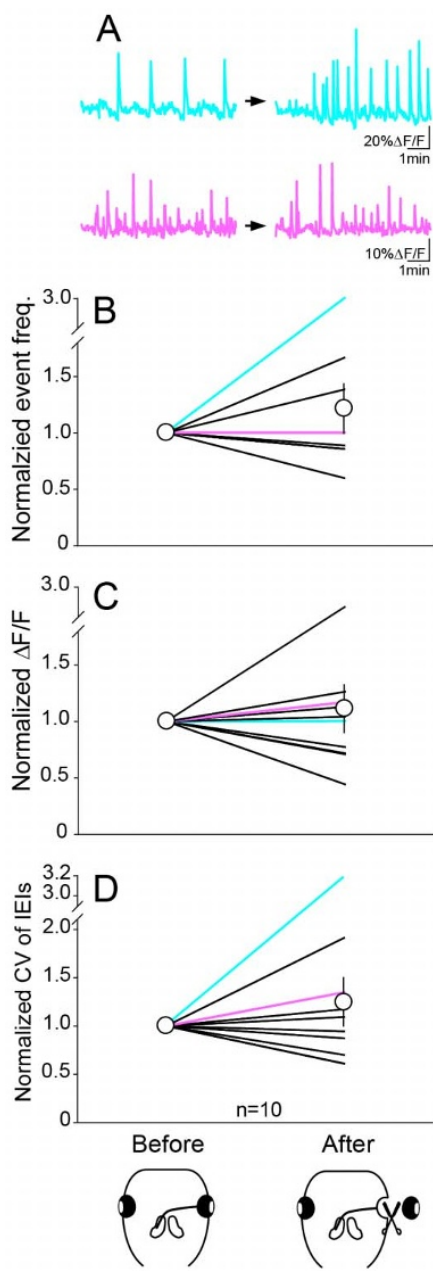

Eye enulceation
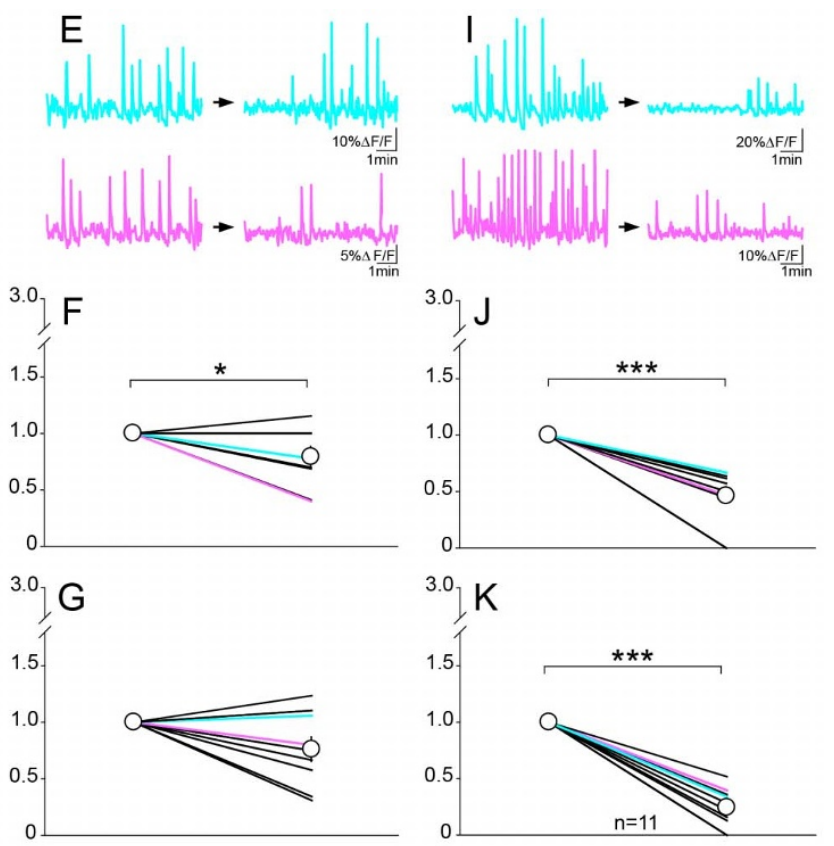

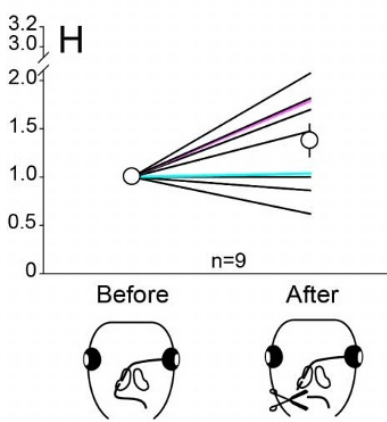

HB connection cut

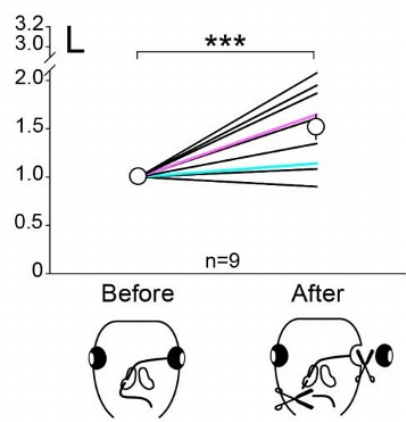

Eye enulceation
HB connection cut

Figure $5 \mid$ Manipulation of input to the optic tectum. (A-D) Effect of enucleating the contralateral eye on spontaneous activity. Spontaneous Ca ${ }^{2+}$ events were assessed in a large area including the cell body layer and the neuropil. (A) Two examples of spontaneous $\mathrm{Ca}^{2+}$ events before (left) and after enucleation (right). (B-D) Event frequency, magnitude, and CV of IEIs of spontaneous activity before and after the enucleation. Data are normalized to the values before the manipulation. Comparisons were performed by paired $t$-tests in 10 tecta. $(\mathrm{E}-\mathrm{H})$ Effect of cutting the connection from the hindbrain to the tectum. (E) Two examples of spontaneous $\mathrm{Ca}^{2+}$ events before (left) and after cutting the connection between the tectum and the hindbrain (right). $(\mathrm{F}-\mathrm{H})$ Event frequency, magnitude, and CVs of IEIs of spontaneous activity before and after cutting the connection. *: $p<0.05$. (I-L) Effect of enucleating the contralateral eye and cutting the connection from the hindbrain to the tectum. (I) Two examples of spontaneous $\mathrm{Ca}^{2+}$ events before (left) and after the paired manipulations (right). (J-L) Event frequency, magnitude, and CVs of IEIs of spontaneous activity before and after the paired manipulations. ***: $p<0.001$. In (L), two cases that lost spontaneous activity after the manipulations were not included. Error bars are S.E.M. Colors in (A, E, and I) correspond to the same tecta in (B-D, F-H, and J-L). HB: hindbrain.

activity of many individual cells. The current study produced three main findings. First, we report the novel finding of global, highly synchronous spontaneous $\mathrm{Ca}^{2+}$ activity, which we refer to as hypersynchronous activity, in in vivo tectal preparations. We analyzed this spontaneous activity in the input and output layers by assessing activity in the neuropil (ascending axons and tectal dendrites; input) and in the tectal somata (output), respectively. Based on the results, we found that although spontaneous activity in the input layer depended on stage but not visual experience, synchronous activity in the output layer depended on both developmental stage and visual experience. Second, we found that while the spontaneous activity is generated intrinsically, the rhythmic patterns result from inputs from retinotectal (visual system) and hindbrain-tectal (mechanosensory system) projections. However, we can not rule out the possibility of contribution from other sources such as the forebrain ${ }^{30}$ and/or the other tectum ${ }^{31}$. However, these structures are less likely the sources providing strong patterned spontaneous input because their anatomical connections are smaller ${ }^{32}$. Third, in sharp contrast to the global hyper-synchronous spontaneous activity between neurons, we found local interactions in spontaneous activity between non-neuronal cells.

An important question is what membrane and spiking properties underlie our spontaneous $\mathrm{Ca}^{2+}$ events. There is strong correlation between $\mathrm{Ca}^{2+}$ events and the number of spikes ${ }^{17,33,34}$. Thus, we propose that we most likely assayed bursts of spikes as evidenced by the longer time constant of the $\mathrm{Ca}^{2+}$ events $(\sim 5 \mathrm{~s})^{7,15}$. Alternatively, our spontaneous $\mathrm{Ca}^{2+}$ events might be spontaneous synaptic barrages observed in in vitro preparations $s^{35}$. Despite the similar occurrence frequency (2.5-4.0/min for spontaneous $\mathrm{Ca}^{2+}$ events vs. $2.1 / \mathrm{min}$ for spontaneous synaptic barrages), however, this is less likely a candidate because spontaneous synaptic barrages occur largely in later developmental stages. In contrast, we observed spontaneous $\mathrm{Ca}^{2+}$ events in all stages of our in vivo preparations.

With regard to our first finding, what may account for spontaneous $\mathrm{Ca}^{2+}$ events in the input layer depending on the developmental 
A
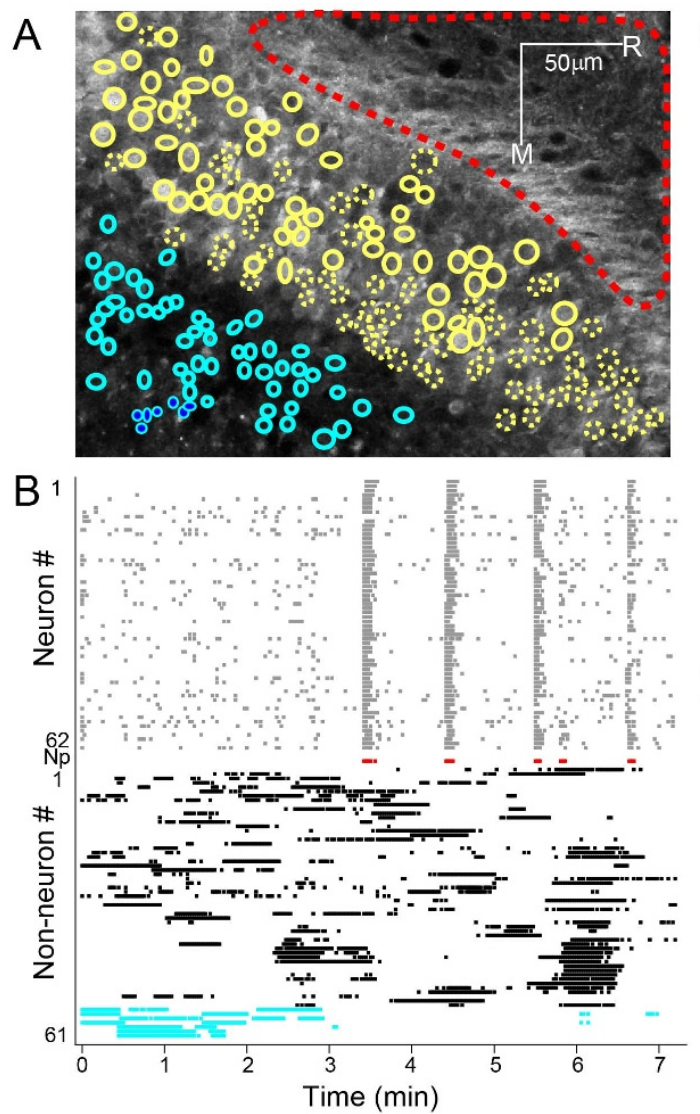

E

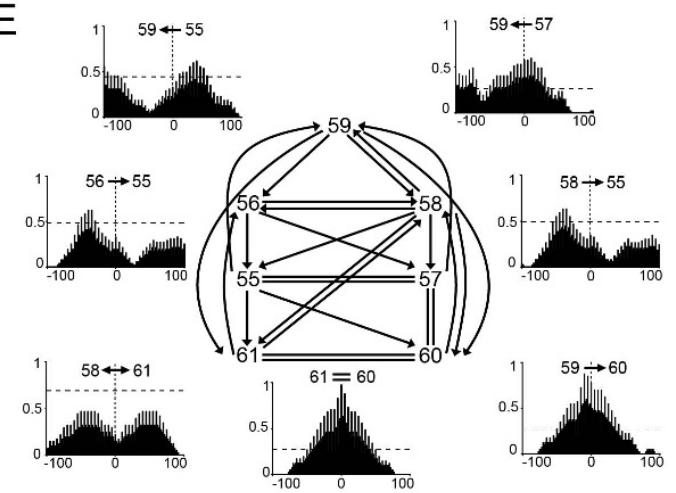

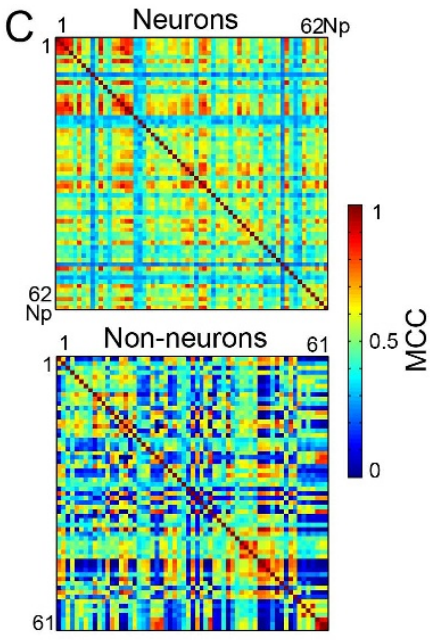
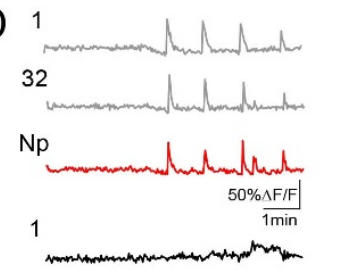

11
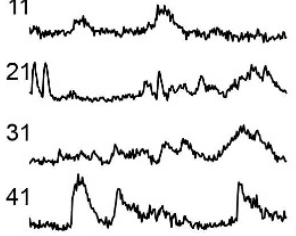

55

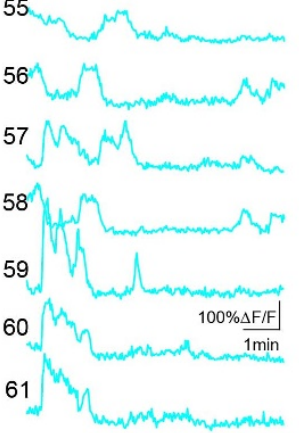

Figure 6 Contrast between local non-neuronal and global neuronal synchronization. (A) ROIs of neurons (yellow circles), non-neuronal cells (blue circles) and neuropil (red dashed line) on average of three movie frames. Yellow dashed circles illustrate ROIs of neurons without spontaneous activity. Blue filled circles represent seven non-neuronal cells used in (B, D, and E). (B) Raster plot of spontaneous $\mathrm{Ca}^{2+}$ events of 62 neurons (gray), the neuropil (red), and 61 non-neuronal cells (black and blue). Note the global structure of spontaneous $\mathrm{Ca}^{2+}$ events in neurons and the neuropil and lack of that in non-neuronal cells. (C) MCC values of CCFs for all pairs of single neurons and the neuropil (top) and non-neuronal cells (bottom). Medians of MCC values: 0.48 for neurons and neuropil, 0.44 for non-neuronal cells. (D) Spontaneous $\mathrm{Ca}^{2+}$ traces of two neurons (gray), the neuropil $(\mathrm{Np})$ (red), and 12 non-neuronal cells (black and blue). Spontaneous $\mathrm{Ca}^{2+}$ traces (blue) in seven non-neuronal cells were analyzed for local interactions. (E) Assessing local interactions among seven non-neuronal cells were performed by CCF analysis. Insets illustrate seven CCFs to show complex interactions.

stage but not on visual experience? One explanation might be in methodology. Whereas short term visual experience enhances tectal dendrite growth ${ }^{18}$, the long term visual experience used in the current study may not have a distinctive effect. Alternatively, it is more likely that spontaneous $\mathrm{Ca}^{2+}$ events in the input layer may derive mainly from ascending axons, not tectal dendrites ${ }^{17,36}$. In a previous study that used genetically encoded $\mathrm{Ca}^{2+}$ sensor, the retinotectal axons in the developing tectum clearly showed spontaneous $\mathrm{Ca}^{2+}$ events between stimulus-evoked activities (movie S2 in supplemental information of Ref. 36). In contrast to the events in the input layer, synchronous spontaneous $\mathrm{Ca}^{2+}$ events in the output layer depended on both stage and visual experience. Because $\mathrm{Ca}^{2+}$ traces of spontaneous activity in the neuropil (input) and somata (output) were quite similar (Fig. 2B, 3, 6D) and tightly correlated (Fig. 4), the different influence of visual experience between input and output layers implicates recurrent activity within the tectum. In the current study, visual experience enhanced synchronous spontaneous $\mathrm{Ca}^{2+}$ events at stage $45 / 46$ and 49/50 (Fig. 2D) with the interesting exception at stage 47/ 48 , when the situation was reversed. Higher synchronous activity (e.g., higher MCC values) at stage 45/46 and 49/50 is presumably 
mediated by tight synaptic coupling via neural connections that is enhanced by sensory experience ${ }^{19}$. However, higher synchronous activity is also caused by the reduction of GABAergic functions ${ }^{37,38}$. In the tectum, both developmental stage and visual experience influence the GABAergic function and expression. GABAergic synaptic conductance decreases from stage $43 / 44$ to $47 / 48^{39}$, and GABA expression is decreased by short term dark exposure at stage $47^{40}$. Stage $47 / 48$ is a transitional period when the amount of excitability (thus, inhibitory to excitatory ratio) varies considerably ${ }^{35}$. Taken together, reduction of GABAergic functions in the dark-reared tecta might have reversed the experience effect, enhancing synchronous spontaneous activity at stage $47 / 48$. In spite of this explanation, it is still not clear why, in the control rearing, neurons at stage $47 / 48$ had lower synchronous spontaneous $\mathrm{Ca}^{2+}$ events than those at other stages.

In a recent study, $\mathrm{Xu}$ et al. (2011) combined in vitro $\mathrm{Ca}^{2+}$ imaging with a fast frame speed (125 fps) and deconvolution techniques, which enabled extrapolation of spike firing rate ${ }^{41}$. With regard to spontaneous activity, their results showed two important differences from ours. First, in vitro preparations showed that spontaneous activity did not depend on visual experience (note that their in vivo preparations did, however, show experience-dependent visual stimulus-evoked activity). Second, with respect to synchronous spontaneous activity, they reported much lower correlation coefficient values $(<0.1)$ than we did here $(\sim 0.5)$. These differences between Xu et al. (2011) and our study can be explained by the fact that they extrapolated spike firing rate, and we most likely assayed bursts of spikes, as discussed above; whereas spontaneous spikes occur more frequently (every 200-500 ms) with seemingly random forms in the tectum ${ }^{35}$, spontaneous $\mathrm{Ca}^{2+}$ events in our study occurred less frequently (every 15-20 s) with more structured rhythmic patterns. Additionally, their use of the in vitro preparations potentially removed input from multisensory systems to the tectum, which we have determined here to be critical to the characteristics of spontaneous activity. Therefore, we believe that the two different studies measured different phenomena.

Our manipulation results shed light on the importance of multisensory integration for spontaneous activity in the developing brain. Rhythmic spontaneous $\mathrm{Ca}^{2+}$ events reported here exhibit some characteristics typical of rhythmic bursting found in a number of other developing brain circuits ${ }^{14}$. IEIs in the rhythmic activity vary from a few sec to $>1 \mathrm{~min}$, which is in a good agreement with our results (15-20 s). In those other systems, intrinsic neural circuits generate rhythmic patterns of spontaneous bursting activity ${ }^{14}$. Our manipulation experiments, however, suggest that either input from retinalganglion or hindbrain-tectal projections generates the rhythmic spontaneous patterns. A recent study that employed in vivo twophoton $\mathrm{Ca}^{2+}$ imaging techniques in the mouse visual system has reported that retinal waves before eye opening control spontaneous activity patterns in both the superior colliculus (a mammalian homologue of the optic tectum) and the primary visual cortex ${ }^{42}$. Elimination of retinal activity completely abolishes spontaneous activity in the superior colliculus. Thus, the mammalian visual system may have developmentally different circuit formation from the Xenopus visual (or multisensory) system, although our experiments were done after establishment of functional vision. In Xenopus tadpoles, the retina by itself does not produce synchronized spontaneous bursting activity ${ }^{43}$. Development of tectal circuitry, thus, is important for the generation of spontaneous busting activity. However, retinotectal (visual) and hindbrain-tectal (mechanosensory) input proceeds in a complex set of steps. Visual and mechanosensory axons reach and intermingle in the tectum as early as at stage $39 / 40^{22,23}$. By stage 48 , however, they have more precise topographic organization by which visual and mechanosensory axons are distributed in the neuropil corresponding to the distal and proximal areas of tectal dendrites, respectively. At this stage, frequency and magnitude of spontaneous $\mathrm{Ca}^{2+}$ events in the neuropil are the highest (Fig. 1). The detailed synaptic maturation and innervations, however, are different between visual and mechanosensory input to the tectum. In the excitatory synapse, the AMPA/NMDA receptor ratio in the visual tectal neurons increases from stage 40 to $48 / 49^{44}$, while that in the mechanosensory tectal neurons decreases from stage 44-46 to $48 / 49^{22}$. Taken together, these data are still insufficient to understand the neural mechanisms of the generation and enhancement of spontaneous activity by either input.

In the future, it is important to study how global hyper-synchronous spontaneous activity is related to topographic organization of physiological properties of tectal neurons $s^{33}$ and, as a model for multisensory development, how topographic projections of retinotectal and hindbrain-tectal systems integrate to enhance spontaneous activity. Our study is a first step toward understanding development of topographic organization related to spontaneous activity in the optic tectum.

\section{Methods}

Animal preparations. All experimental protocols were approved by LSUHSC-NO Institutional Animals Care and Use Committee in accordance with federal guidelines. Embryonic albino tadpoles of Xenopus laevis were purchased (Xenopus Express, Brooksville, FL), and raised to an appropriate stage ${ }^{45}$ in $10 \%$ Steinberg's solution (100\% Steinberg's solution: $58.2 \mathrm{mM} \mathrm{NaCl}, 0.67 \mathrm{mM} \mathrm{KCl}, 0.34 \mathrm{mM} \mathrm{Ca}\left(\mathrm{NO}_{3}\right)_{2}$, $0.78 \mathrm{mM} \mathrm{MgSO}_{4}, 10 \mathrm{mM}$ HEPES, pH 7.4) in an aquarium $(12 \mathrm{~cm} \times 18 \mathrm{~cm} \times$ $14 \mathrm{~cm}$ ) in a 12 hours light/dark cycle. For dark-reared experiments, tadpoles were kept in the dark from stage 41/42 until the day of experiments (2-3 days for stage 45/ 46, 3-7 days for stage 47/48, and 10-14 days for stage 49/50). The rearing solution was changed (3-4 times/week) under red light. Tadpoles were anesthetized with $0.02 \%$ MS-222 (Sigma-Aldrich, St Louis, MO) in saline: $115 \mathrm{mM} \mathrm{NaCl}, 2 \mathrm{mM} \mathrm{KCl}$, $2.5 \mathrm{mM} \mathrm{CaCl}_{2}, 1 \mathrm{mM} \mathrm{MgCl}_{2}, 10 \mathrm{mM}$ glucose, $10 \mu \mathrm{M}$ glycine, $10 \mathrm{mM}$ HEPES $(\mathrm{pH} 7.3)^{34}, 252 \mathrm{mOsm}$ and secured by three insect pins on a Sylgard dish. Skin over the midbrain was removed. During the surgery, tadpoles for both the control and dark-reared groups were exposed to white light for a few min.

Calcium indicator loading. Cell-permeant intracellular calcium indicator $(0.8 \mathrm{mM}$ OGB1-AM, 2\% Pluronic acid, 10\% DMSO, $40 \mu \mathrm{M}$ Alexa Fluor 594, all from Invitrogen (Carlsbad, CA)) was prepared in $\mathrm{Ca}^{2+}$ free saline, and filtered by a $0.22 \mu \mathrm{m}$ centrifugal filter tube (Millipore, Billerica, MA). Using a glass pipette (tip diameter of $\sim 5 \mu \mathrm{m}$ ) advanced into the optic tectum, the OGB1-AM solution was pressureinjected into the tectum (Picospritzer II, Parker Hannifin, Cleveland, $\mathrm{OH}$ ) at 5-20 psi multiple times over several min (1-2 s/injection $)^{46}$. The injections were monitored by two-photon microscope (see below) with a $610 \mathrm{~nm}$ emission filter (HQ610/75m-2p, Chroma Technology, Bellows Falls, VT). Subsequently, tadpoles were incubated in the fresh oxygen-saturated saline under dim light condition for $1 \mathrm{hr}$ and recovered from the anesthesia at room temperature ${ }^{33}$. To immobilize the brain and body movement, tadpoles were immersed in $10 \mathrm{mM}$ d-tubocurarine (Sigma-Aldrich) for 2-3 min, washed by the oxygen-saturated saline, and embedded in $1.4 \%$ low-melting point agarose (Sigma-Aldrich) prepared in saline ${ }^{47}$. During the $\mathrm{Ca}^{2+}$ imaging, the oxygen-saturated saline was continuously perfused. All experiments were performed at room temperature. During preliminary experiments, we performed similar experiments without $10 \mathrm{mM}$ d-tubocurarine and $1.4 \%$ agarose, and obtained the same global synchronous spontaneous activity.

In vivo two-photon $\mathrm{Ca}^{2+}$ imaging. The custom-made imaging chamber was attached to an Olympus FV 300 two-photon microscope (Olympus, Tokyo, Japan) and titanium: sapphire laser (Chameleon Ultra-II, Coherent, Santa Clara, CA). Neuronal and "non-neuronal" activity was monitored at 40-150 $\mu \mathrm{m}$ depth using a water immersion objective lens (Olympus XLUMPlanF1, 20x, $0.95 \mathrm{NA}$ ) at $820 \mathrm{~nm}$ wavelength with a $540 \mathrm{~nm}$ emission filter (HQ540/40m-2p, Chroma Technology) and detected with a photomultiplier tube (Hamamatsu Photonics, Hamamatsu, Japan). Imaging data were acquired by Fluoview FV300 software (Olympus). For single-cell resolution imaging, a zoom factor of 3-4× was used at a resolution of 800 $\times 600$ pixels. Each frame was scanned at a $1.67 \mathrm{~s}$ speed $(0.6 \mathrm{fps})$. For faster scanning (10-100 fps), the frame size was adjusted ( $512 \times 12-92$ pixels). Each imaging session at $0.6 \mathrm{fps}$ lasted for 6-8 min (a standard session was made for $7 \mathrm{~min} 15 \mathrm{~s}$ with 260 frames). The faster imaging sessions lasted for $1 \mathrm{~min} 30 \mathrm{~s}$ to $3 \mathrm{~min} 20 \mathrm{~s}$. Longer imaging sessions were discarded because of $\mathrm{z}$-drift.

Manipulation of spontaneous input from sensory systems. To investigate the source of spontaneous activity in the optic tectum, we eliminated input from the visual and/or mechanosensory system by enucleating a contralateral eye and/or cutting the connection between the optic tectum and the hindbrain, respectively. A $30 \mathrm{G}$ needle was inserted into the contralateral eye socket and smoothly removed an eye and/or was deeply inserted and moved along the posterior edge of the ipsilateral optic tectum (same side as imaged). When the manipulations caused edema in the tectum, the data were discarded. 
Data analysis. Imaging data were aligned using Turboreg ${ }^{48}$. The imaging data with $\mathrm{z}$ drift were discarded. Due to the densely packed cell bodies, ROIs were manually and conservatively selected using Image J (National Institutes of Health). Fluorescence intensity changes were computed as $\Delta \mathrm{F} / \mathrm{F}$, in which $\Delta \mathrm{F}$ is the difference between the average intensity of ROI and the baseline intensity at each frame and F is the average intensity of ROI across all frames after the baseline subtraction. The baseline was computed by a third-order nonparametric, local regression fit ${ }^{49}$. The vicinity of weighting was determined empirically (typically $0.2-0.4$ ). Fluorescent change that occurred above $1 \mathrm{SD}$ was deemed a $\mathrm{Ca}^{2+}$ event. For imaging data of non-neuronal cells, the same procedures were used without a baseline correction. For manipulation experiments, a 2 SD criterion was conservatively used. However, the normalized results with a 2 SD criterion before and after the manipulations had a similar trend to those with a $1 \mathrm{SD}$ criterion. Cross correlation analysis of $\mathrm{Ca}^{2+}$ events between pairs of cells was performed by MATLAB (MathWorks, Natick, MA). The significance of the largest peak of each CCF (termed maximum correlation coefficient, MCC, in Fig. 2) was determined by a $99 \%$ confidence threshold. This threshold was defined by fitting a $t$-distribution to the correlation coefficient values at lags longer than $50 \mathrm{~s}$ and finding the upper $99 \%$ confidence cutoff point, adjusted for multiple comparisons by a Bonferroni correction. For the normal imaging data sets ( $0.6 \mathrm{fps})$, a $1 \mathrm{~s}$ bin resolution was used. For the faster imaging data sets (10-100 fps), bin resolution was the same as the imaging speeds. Results were considered when CCFs had a peak within a \pm 50 s time lag. For a statistical comparison of median MCC values (e.g., Fig. 2D), a resampling test was performed because single somata are involved in multiple MCC values and, thus, MCC values from MCC matrices (e.g., Fig. $2 \mathrm{C}$ ) are not independent. To address this issue, we randomly selected MCC values from each MCC matrix such that the neuropil and each soma were involved in at most one MCC value, thus creating an independently sampled subset of MCC values. We used these independent samples to test the null hypothesis that there was no difference in the median MCC values between two groups. We performed this test by comparing the difference in medians between the two independent samples with the difference in medians obtained if we shuffled the samples between the two groups. We performed this sampling and testing procedure 10,000 times for each resampling test. All other statistical analyses were performed using StatView (SAS Institute, Cary, NC).

1. Cang, J. et al. Development of precise maps in visual cortex requires patterned spontaneous activity in the retina. Neuron 48, 797-809 (2005).

2. Katz, L. C. \& Shatz, C. J. Synaptic activity and the construction of cortical circuits. Science 274, 1133-1138 (1996)

3. Ch'ng, Y. H. \& Reid, R. C. Cellular imaging of visual cortex reveals the spatial and functional organization of spontaneous activity. Front Integr Neurosci 4, doi:10.3389/fnint.2010.00020 (2010).

4. Kenet, T., Bibitchkov, D., Tsodyks, M., Grinvald, A. \& Arieli, A. Spontaneously emerging cortical representations of visual attributes. Nature 425, 954-956 (2003)

5. Tsodyks, M., Kenet, T., Grinvald, A. \& Arieli, A. Linking spontaneous activity of single cortical neurons and the underlying functional architecture. Science 286, 1943-1946 (1999).

6. Golshani, P. et al. Internally mediated developmental desynchronization of neocortical network activity. J Neurosci 29, 10890-10899 (2009).

7. Warp, E. et al. Emergence of patterned activity in the developing zebrafish spinal cord. Curr Biol 22, 93-102 (2012).

8. Ruthazer, E. S. \& Cline, H. T. Insights into activity-dependent map formation from the retinotectal system: a middle-of-the-brain perspective. J Neurobiol 59, 134-146 (2004).

9. Debski, E. A. \& Cline, H. T. Activity-dependent mapping in the retinotectal projection. Curr Opin Neurobiol 12, 93-99 (2002).

10. Cohen-Cory, S. \& Lom, B. Neurotrophic regulation of retinal ganglion cell synaptic connectivity: from axons and dendrites to synapses. Int J Dev Biol $\mathbf{4 8}$, 947-956 (2004).

11. Holt, C. E. \& Harris, W. A. Position, guidance, and mapping in the developing visual system. J Neurobiol 24, 1400-1422 (1993).

12. Deeg, K. E. \& Aizenman, C. D. Sensory modality-specific homeostatic plasticity in the developing optic tectum. Nat Neurosci 14, 548-550 (2011).

13. Xu, H., Khakhalin, A. S., Nurmikko, A. V. \& Aizenman, C. D. Visual experiencedependent maturation of correlated neuronal activity patterns in a developing visual system. J Neurosci 31, 8025-8036 (2011)

14. Blankenship, A. G. \& Feller, M. B. Mechanisms underlying spontaneous patterned activity in developing neural circuits. Nat Rev Neurosci 11, 18-29 (2010).

15. Marder, E. \& Bucher, D. Understanding circuit dynamics using the stomatogastric nervous system of lobsters and crabs. Annu Rev Physiol 69, 291-316 (2007).

16. Siegel, F., Heimel, J. A., Peters, J. \& Lohmann, C. Peripheral and central inputs shape network dynamics in the developing visual cortex in vivo. Curr Biol 22, 253-258 (2012).

17. Kerr, J. N., Greenberg, D. \& Helmchen, F. Imaging input and output of neocortical networks in vivo. Proc Natl Acad Sci U S A 102, 14063-14068 (2005).

18. Sin, W. C., Haas, K., Ruthazer, E. S. \& Cline, H. T. Dendrite growth increased by visual activity requires NMDA receptor and Rho GTPases. Nature 419, 475-480 (2002).

19. Pratt, K. G., Dong, W. \& Aizenman, C. D. Development and spike timingdependent plasticity of recurrent excitation in the Xenopus optic tectum. Nat Neurosci 11, 467-475 (2008)
20. Zhang, L. I., Tao, H. W., Holt, C. E., Harris, W. A. \& Poo, M. A critical window for cooperation and competition among developing retinotectal synapses. Nature 395, 37-44 (1998).

21. Ruthazer, E. S. \& Aizenman, C. D. Learning to see: patterned visual activity and the development of visual function. Trends Neurosci 33, 183-192 (2010).

22. Deeg, K. E., Sears, I. B. \& Aizenman, C. D. Development of multisensory convergence in the Xenopus optic tectum. J Neurophysiol 102, 3392-3404 (2009)

23. Hiramoto, M. \& Cline, H. T. Convergence of multisensory inputs in Xenopus tadpole tectum. Dev Neurobiol 69, 959-971 (2009).

24. Tremblay, M. et al. Regulation of radial glial motility by visual experience. J Neurosci 29, 14066-14076 (2009).

25. Bestman, J. E., Lee-Osbourne, J. \& Cline, H. T. In vivo time-lapse imaging of cell proliferation and differentiation in the optic tectum of Xenopus laevis tadpoles. J Comp Neurol 520, 401-433 (2012).

26. Buzsaki, G. \& Draguhn, A. Neuronal oscillations in cortical networks. Science 304, 1926-1929 (2004)

27. Fries, P. A mechanism for cognitive dynamics: neuronal communication through neuronal coherence. Trends Cog Sci 9, 474-480 (2005).

28. Varela, F., Lachaux, J. P., Rodriguez, E. \& Martinerie, J. The brainweb: phase synchronization and large-scale integration. Nat Rev Neurosci 2, 229-239 (2001).

29. Wang, X. J. Neurophysiological and computational principles of cortical rhythms in cognition. Physiol Rev 90, 1195-1268 (2010).

30. Marin, O., Smeets, W. J. \& Gonzalez, A. Evolution of the basal ganglia in tetrapods: a new perspective based on recent studies in amphibians. Trends Neurosci 21, 487-494 (1998).

31. Herrero, L., Perez, P., Nunez Abades, P., Hardy, O. \& Torres, B. Tectotectal connectivity in goldfish. J Comp Neurol 411, 455-471 (1999).

32. Horowitz, S. S. \& Simmons, A. M. Development of tectal connectivity across metamorphosis in the bullfrog (Rana catesbeiana). Brain Behav Evo 76, 226-247 (2010).

33. Niell, C. M. \& Smith, S. J. Functional imaging reveals rapid development of visual response properties in the zebrafish tectum. Neuron 45, 941-951 (2005).

34. Bollmann, J. H. \& Engert, F. Subcellular topography of visually driven dendritic activity in the vertebrate visual system. Neuron 61, 895-905 (2009).

35. Pratt, K. G. \& Aizenman, C. D. Homeostatic regulation of intrinsic excitability and synaptic transmission in a developing visual circuit. J Neurosci 27, 8268-8277 (2007).

36. Nikolaou, N. et al. Parametric functional maps of visual inputs to the tectum. Neuron 76, 317-324 (2012).

37. Klueva, J., Meis, S., de Lima, A. D., Voigt, T. \& Munsch, T. Developmental downregulation of GABAergic drive parallels formation of functional synapses in cultured mouse neocortical networks. Dev Neurobiol 68, 934-949 (2008).

38. El-Hassar, L. et al. Cell domain-dependent changes in the glutamatergic and GABAergic drives during epileptogenesis in the rat CA1 region. J Physiol 578, 193-211 (2007)

39. Tao, H. W. \& Poo, M. M. Activity-dependent matching of excitatory and inhibitory inputs during refinement of visual receptive fields. Neuron 45, 829-836 (2005).

40. Miraucourt, L. S. et al. GABA Expression and Regulation by Sensory Experience in the Developing Visual System. PLoS One 7, e29086 (2012).

41. Yaksi, E. \& Friedrich, R. W. Reconstruction of firing rate changes across neuronal populations by temporally deconvolved $\mathrm{Ca}^{2+}$ imaging. Nat Methods 3, 377-383 (2006).

42. Ackman, J. B., Burbridge, T. J. \& Crair, M. C. Retinal waves coordinate patterned activity throughout the developing visual system. Nature 490, 219-225 (2012).

43. Demas, J. A., Payne, H. \& Cline, H. T. Vision drives correlated activity without patterned spontaneous activity in developing Xenopus retina. Dev Neurobiol 72, 537-546 (2012)

44. Akerman, C. J. \& Cline, H. T. Depolarizing GABAergic conductances regulate the balance of excitation to inhibition in the developing retinotectal circuit in vivo. J Neurosci 26, 5117-5130 (2006).

45. Niewkoop, P. D. \& Faber, J. Normal Table of Xenopus laevis (Daudin). (Elsevier North-Holland, 1956).

46. Brustein, E., Marandi, N., Kovalchuk, Y., Drapeau, P. \& Konnerth, A. "In vivo" monitoring of neuronal network activity in zebrafish by two-photon $\mathrm{Ca}^{2+}$ imaging. Pflugers Arch 446, 766-773 (2003).

47. Dunfield, D. \& Haas, K. Metaplasticity governs natural experience-driven plasticity of nascent embryonic brain circuits. Neuron 64, 240-250 (2009).

48. Thevenaz, P., Ruttimann, U. E. \& Unser, M. A pyramid approach to subpixel registration based on intensity. IEEE Trans Image Process 7, 27-41 (1998).

49. Sumbre, G., Muto, A., Baier, H. \& Poo, M. M. Entrained rhythmic activities of neuronal ensembles as perceptual memory of time interval. Nature 456, 102-106 (2008).

\section{Acknowledgments}

We thank Hiroki Sugihara and Kenichi Ohki for technical advice, Craig Atencio for statistical advice, and Andrew Bass, Bruce Carlson, Cristopher Niell, and Andrew Tan for comments. This study was supported by NIH grant P20RR016816 to Nicolas Bazan. KI was supported by USDA Competitive Research Program Funds (LAV-3300). 


\section{Author contributions}

K.I. designed and performed experiments, K.I., J.Y.S. and H.E.F. analyzed data, J.Y.S. contributed analytical tools, H.E.F. and K.I. maintained two-photon microscope, K.I., J.Y.S. and H.E.F. wrote manuscript.

\section{Additional information}

Supplementary information accompanies this paper at http://www.nature.com/ scientificreports
Competing financial interests: The authors declare no competing financial interests. License: This work is licensed under a Creative Commons

Attribution-NonCommercial-ShareALike 3.0 Unported License. To view a copy of this license, visit http://creativecommons.org/licenses/by-nc-sa/3.0/

How to cite this article: Imaizumi, K., Shih, J.Y. \& Farris, H.E. Global Hyper-synchronous Spontaneous Activity in the Developing Optic Tectum. Sci. Rep. 3, 1552; DOI:10.1038/ srep01552 (2013). 